\title{
Effect of internal heat generation or absorption on MHD mixed convection flow in a lid driven cavity
}

\author{
Litan Kumar Saha ${ }^{1}$, K. M. Salah Uddin ${ }^{2}$, M. A. Taher ${ }^{3}$ \\ ${ }^{1}$ Department of Applied Mathematics, University of Dhaka, Dhaka, Bangladesh \\ ${ }^{2}$ Department of Management Information Systems, University of Dhaka, Dhaka, Bangladesh \\ ${ }^{3}$ Deparment of Mechanical and Automotive Engineering, Pukyong National University, Busan, Korea
}

\section{Email address:}

lksaha.math@gmail.com (L. K. Saha), salahuddin@du.ac.bd (K. M. S. Uddin), taher@pknu.ac.kr (M. A. Taher)

\section{To cite this article:}

Litan Kumar Saha, K. M. Salah Uddin, M. A. Taher. Effect of Internal Heat Generation or Absorption on MHD Mixed Convection Flow in a Lid Driven Cavity. American Journal of Applied Mathematics. Special Issue: Fluid Flow and Heat Transfer Inside a Closed Domain. Vol. 3, No. 1-1, 2015, pp. 20-29. doi: 10.11648/j.ajam.s.2015030101.13

\begin{abstract}
In the present study the problem of mixed convection flow in the presence of magnetic field in a lid-driven square cavity with internal heat generation or absorption and uniform heating of bottom wall were investigated numerically. The square cavity vertical walls are maintained at cold temperature while the upper wall is insulated. The physical problem is then expressed mathematically by a set of governing equations and the developed mathematical model is solved by employing Galerkin weighted residual method of finite element formulation. Effects of variations of Richardson number, Hartmann number and heat generation or absorption parameter on flow structure and heat transfer rate (Nuesselt number) were studied in details. The significant reduction in the average Nusselt number were produced as the strength of the applied magnetic field was increased. In addition, heat generation predicted to decrease the average Nusselt number whereas heat absorption increases it.
\end{abstract}

Keywords: Mixed Convection, Lid Driven Cavity, Heat Transfer, Numerical Study, Heat Generation/Absorption

\section{Introduction}

Mixed convection flow and heat transfer in a lid-driven enclosure has attracted considerable attention due to its ap-plications, especially those related to lubrication technologies, electronic cooling, food processing and nuclear reactors [1-2]. The influence of the magnetic field on the mixed convection flow of the fluid is of paramount importance in industrial engineering. A combined free and forced convection flow of an electrically conducting fluid in a cavity in the presence of magnetic field is of special technical significance because of its frequent occurrence in many industrial applications such as geothermal reservoirs, cooling of nuclear reactors, thermal insulations and petroleum reservoirs. These types of problems also arise in electronic packages, microelectronic devices during their operations [3]. There are many experimental and numerical investigations are found in the literature on lid-driven cavity flow and heat transfer during the past three decades. Mohamad and Viskanta [4] studied the influence of a sliding lid on the flow structures and heat transfer in a shallow lid-driven cavity. Their study shows that that the maximum local heat transfer rate occurs at the start-ing area of the sliding lid and decreases along the sliding lid. Tillmark [5] conducted an experimental and numerical study on the lid-driven flow in a polar cavity. Migeon et al. [6] in-vestigated experimentally the effects of lid-driven cavity shape on the flow establishment phase for square, rectan-gular and semi-circular cavities. Moallemi and Jang [7] studied numerically mixed convective flow in a bottom heated square cavity. They found that the effects of buoyancy are more pronounced for higher values of Prandtl number square lid-driven cavity. Al-Amiri et al. [8] studied a numerical investigation of mixed convection flow in lid-driven cavity with a sinusoidal wavy bottom surface. Their results concluded that the corrugated lid-driven cavity could be considered as an effective heat transfer mechanism at larger wavy surface amplitudes. Cheng and coworkers [9-11] investigated the flow structures and heat transfer behaviors for mixed convective flow within lid driven arc-shaped enclosures of varying inclination angle with different thermal gradients. Saha et al. [12] investigated the mixed convection flow and heat transfer in a lid-driven cavity with wavy bottom surface and the governing equations 
are solved using the Galerkin finite element method.. Oztop et al. [13] examined mixed convection heat transfer charac-teristics for a lid-driven air flow within a square enclosure having a circular body. They found that the circular body has significant effect on flow field and temperature distribution.

Mixed convection in porous cavity has also been studied in presence of internal heat generation. Khanafer and Chamkha [14] investigated the laminar, mixed convection flow in a Darcian fluid-saturated porous cavity with internal heat generation and they observed that the flow and the heat transfer inside the cavity were strong function of Richardson number (Ri). The effect of surface tension on unsteady laminar natural convection flow of an electrically conducting fluid in a rectangular enclosure under an extremely imposed magnetic field with internal heat generation has been investigated by Hossain et al. [15]. Mahapatra et al. [16] studied the mixed convection problem in differentially heated square enclosure with two adiabatic partitions. Their results showed that the effect of the partition location on heat transfer is marginal for $\mathrm{Ri}=1.0$ and which is more pronounced for $\mathrm{Ri}=0.1$. Sivakumar et al. [17] numerically analyzed the mixed convection heat transfer and fluid flow in lid-driven cavities where the left vertical wall is partially heated. Iwatsu et al[18] analyzed numerically mixed convection heat transfer in a driven cavity with a stable vertical temperature gradient. Their results have revealed that the flow features are similar to those of a conventional lid driven cavity of a non-stratified fluid for small values of the Richardson number. Basak et al. [19] investigated the influence of uniform and non-uniform heating of bottom wall while vertical walls are maintained at cold temperature on mixed convection lid driven cavity flows in a square cavity. Their results showed that the heat transfer rate is very high at the edges of bottom wall and then decreases at the center of the bottom wall for uniform heating and contrast lower heat transfer rate at the edges for the non-uniform heating of the bottom wall.

Chandra and Gosh [20] investigated the effect of magnetic field on electrically conducted visco-elastic fluid; they found in such a flow that the velocity field increases with decrease of magnetic field strength. Chamkha [21] made an analysis for mixed convection in a square cavity in the presence of the magnetic field and an internal heat generation or absorption. His study found revealed that flow behavior and the heat transfer characteristics inside the cavity are strongly affected by the presence of the magnetic field. Garandet et al. [22]studied natural convection heat transfer in a rectangular enclosure with a transverse magnetic field. The problem of conjugate effect of joule heating and magnato-hydrodynamics mixed convection in an obstructed lid driven was investigated by Rahman et al. [23]. In their study, Galerkin finite element formulation was used. They found that the heat transfer decreases with decreasing of Hartmann number. Laminar mixed convection flow in the presence of magnetic field in a top sided lid-driven heated cavity was investigated by Oztop et al. [24].And, other simi-lar studies can be found in literature as Rudraiah et al. [25], Al-Najem et al. [26], and Kahveci and Oztuna [27].

In all the previous studies, the convection flow behaviors and the heat transfer in cavities with varying thermal bound-aries of uniformity or non-uniformity and the effects of magnetic fields are discussed individually in detail. In overall, all the above studies can be divided into three groups. The first group deals with the mixed convection [3-13] within a cavivities, the second group consists of cavities with various thermal boundary conditions [14-19] and the third group takes magneto convection into consideration [20-27]].

To the best of the authors' knowledge, no attention has been paid to the problem of hydromagnetic mixed convection flow and heat transfer in a lid-driven cavity with internal heat generation.

The main objective of this work is to present the effects of the magnetic field and generation or absorption on mixed convective flow of electrically conducting fluids for a lid-driven cavity with lower wall uniformly heated and the upper wall is insulated. The vertical walls are maintained at cold temperature.

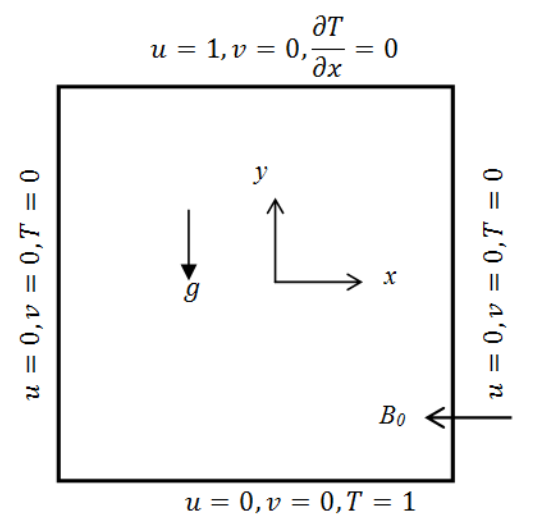

Figure 1. Cavity geometry and boundary condition.

\section{Mathematical Formulation}

A two-dimensional square cavity is considered for the present study with the physical dimension as shown in Fig. 1. The upper wall of the cavity is allowed to move in its own plane at a constant speed $U_{0}$ and the other walls have no-slip condition. The cavity bottom wall is maintained at a high temperature, $T h$, to induce buoyancy effect and the upper wall is insulated. The two vertical walls are maintained at cold temperature, $T c$. The cavity bottom wall is kept at a low temperature, $T c$, and the upper wall is kept at a high temperature, Th. The fluid is assumed to be electrically conducting, all walls of the cavity are assumed to be electrically insulating. A uniform magnetic field with a constant magnitude $B_{0}$ in the direction of the moving lid is applied. It is assumed that the induced magnetic field produced by the motion of an electrically conducting fluid is negligible compared to the applied magnetic field. This assumption uncouples the Navier-Stokes equations from Maxwell's equations. No electric field is present and the Hall effect is neglected. The thermo-physical properties of the 
fluid are assumed to be constant, except the density variation in the buoyancy term which is treated according to Boussinesq approximation while viscous dissipation effects are considered negligible. In general, the cavity fluid is assumed to be Newtonian and incompressible, unsteady and laminar flow.

With abovementioned assumptions, the governing equations for conservations of mass, momentum and energy can be written as

Mass conservation equation:

$$
\frac{\partial u}{\partial x}+\frac{\partial v}{\partial y}=0
$$

Momentum conservation equation:

$$
\begin{gathered}
u \frac{\partial u}{\partial x}+v \frac{\partial u}{\partial y}=-\frac{1}{\rho} \frac{\partial p}{\partial x}+v\left(\frac{\partial^{2} u}{\partial x^{2}}+\frac{\partial^{2} u}{\partial y^{2}}\right) \\
u \frac{\partial v}{\partial x}+v \frac{\partial v}{\partial y}=-\frac{1}{\rho} \frac{\partial p}{\partial y}+v\left(\frac{\partial^{2} v}{\partial x^{2}}+\frac{\partial^{2} v}{\partial y^{2}}\right)+g \beta\left(T-T_{c}\right)-\frac{\sigma B_{0}^{2}}{\rho} v
\end{gathered}
$$

Energy conservation equation:

$$
u \frac{\partial T}{\partial x}+v \frac{\partial T}{\partial y}=\alpha\left(\frac{\partial^{2} T}{\partial x^{2}}+\frac{\partial^{2} T}{\partial y^{2}}\right)+\frac{Q_{0}}{\rho C_{p}}\left(T-T_{c}\right)
$$

where $u$ and $v$ the fluid velocity components in the $x$ - and $y$ directions, $p$ the pressure, $x, y$ the coordinate directions, $T$ the temperature, $\beta$ the volumetric thermal expansion coefficient, $\rho$ the density, $\sigma$ the electrical conductivity and $\alpha$ the thermal diffusivity of the fluid. The parameters $g, B_{0}$ and $Q_{0}$ are the acceleration due to gravity, magnetic induction, and the volumetric internal heat generation $\left(Q_{0}>0\right)$ or absorption $\left(Q_{0}<0\right)$ coefficient, respectively.

The governing equations (1)-(4) are non-dimensionalized using the following dimensionless variables:

$$
X=\frac{x}{L}, Y=\frac{y}{L}, U=\frac{u}{U_{0}}, V=\frac{v}{U_{0}}, P=\frac{p}{\rho U_{0}^{2}}, \theta=\frac{T-T_{c}}{T_{h}-T_{c}}
$$

Introducing the above non-dimensional scales into the governing equations, we obtain the non-dimensional form of the equations as follows:

$$
\begin{aligned}
& \frac{\partial U}{\partial X}+\frac{\partial U}{\partial Y}=0 \\
& U \frac{\partial U}{\partial X}+V \frac{\partial U}{\partial Y}=-\frac{\partial P}{\partial X}+\frac{1}{\operatorname{Re}}\left(\frac{\partial^{2} U}{\partial X^{2}}+\frac{\partial^{2} U}{\partial Y^{2}}\right) \\
& U \frac{\partial V}{\partial X}+V \frac{\partial V}{\partial Y}=-\frac{\partial P}{\partial Y}+\frac{1}{\operatorname{Re}}\left(\frac{\partial^{2} V}{\partial X^{2}}+\frac{\partial^{2} V}{\partial Y^{2}}\right)+\frac{G r}{\operatorname{Re}^{2}} \theta-\frac{H a^{2}}{\operatorname{Re}} v
\end{aligned}
$$

$$
U \frac{\partial \theta}{\partial X}+V \frac{\partial \theta}{\partial Y}=\frac{1}{\operatorname{Re} \operatorname{Pr}}\left(\frac{\partial^{2} \theta}{\partial X^{2}}+\frac{\partial^{2} \theta}{\partial Y^{2}}\right)+\frac{\Delta}{\operatorname{RePr}} \theta
$$

In the above equations $\operatorname{Re}=\frac{U_{0} L}{v}, \operatorname{Pr}=\frac{v}{\alpha}, H a^{2}=\frac{\sigma B_{0}^{2} L^{2}}{\mu}$, $G r=\frac{g \beta \Delta T L^{3}}{v^{2}} \quad$ and $\quad \Delta=\frac{Q_{0} L^{2}}{\alpha \rho C_{p}} \quad$ are respectively the non-dimensional Reynolds number, Prandtl number, Hartmann number, Grashof number and heat generation or absorption coefficient.

The dimensionless boundary conditions are:

$$
\begin{aligned}
& U=1, V=0, \frac{\partial \theta}{\partial X}=0 \quad \text { for } 0 \leq X \leq 1, Y=1 \\
& U=V=0, \theta=1 \quad \text { for } 0 \leq X \leq 1, Y=0 \\
& U=V=0, \theta=0 \text { for } 0 \leq Y \leq 1, X=0 \& X=1
\end{aligned}
$$

Since the convective heat transfer coefficient: $h$ and Nusselt number $\mathrm{Nu}$, depend on the temperature gradient at a flat surface, $\left(-\frac{\partial T}{\partial n}\right)$ where $n$ is normal direction to the surface. Therefore, we can obtain the rate of heat flux from each of the walls. The corresponding average Nusselt number at the heated wall is defined as:

$$
N u_{a v}=\int_{0}^{1} N u d x
$$

\section{Numerical Method and Code Validation}

The non-dimensional equations Eqs. (6) to (9) along with the boundary conditions (10) are solved by utilizing the Galerkin weighted residual method of finite element formulation. The formulation of this method and computational procedure is well described by Taylor and Hood [10] and Dechaumphai [11]. The computational domain is discretized employing the uniform mapped mesh grid system. Then the Galerkin weighted residual technique is used to convert the nonlinear governing partial differential equations into a system of integral equations that can be solved numerically. The integration involved in each term of these equations is performed by using Gauss's quadrature method which leads to a set of non-linear algebraic equations. These equations are then modified by imposing boundary equations that is transferred into linear algebraic equations by Newton-Raphson iteration. Finally, these linear equations are solved by applying Triangular Factorization method.

In order to verify the accuracy of the numerical results obtained throughout the present study is verified against numerical studies reported by Basak et al. [13]. In absence of the magnetic $(\mathrm{Ha}=0)$ and heat generation or absorption $(\Delta=$ 0 ) effects our present model is consistent with those of Basak et al. [13]. The comparison for the contour maps of 
temperature and streamlines are presented in Figures 2 for Re $=1$ and $\mathrm{Gr}=10^{3}, 10^{4}$ and $10^{5}$. It is clear that excellent agreement between the present numerical solutions and those of Basak et al. [13] exists.

$\mathrm{Gr}=10^{4}$
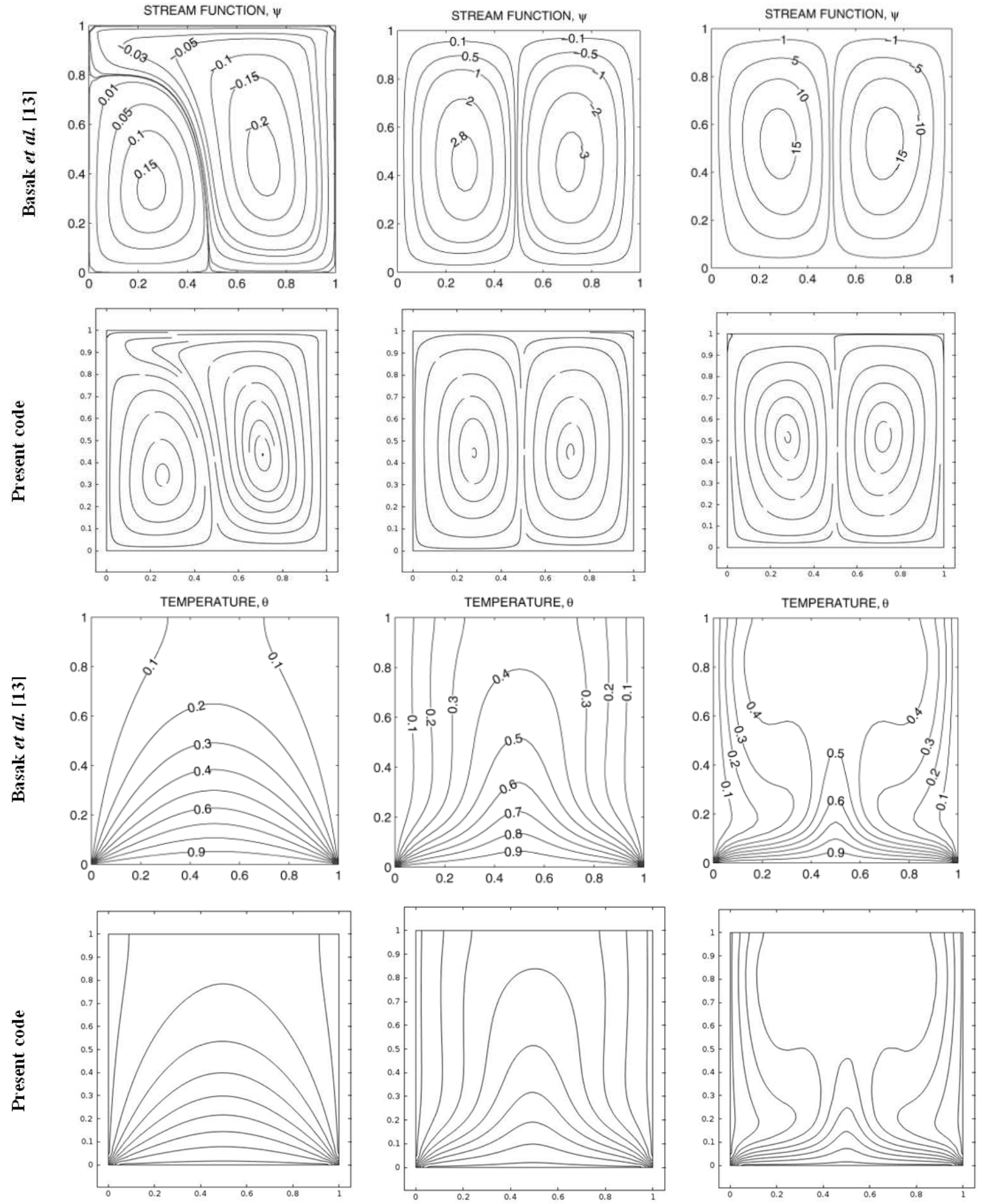

Fig. 2. Comparison of streamlines contour and temperature contour obtained by present code and Basak et al. [13] for $\mathrm{Ha}=0, \Delta=0, \mathrm{Re}=1, \mathrm{Gr}=10^{3}, 10^{4}$ and $10^{5}$ 

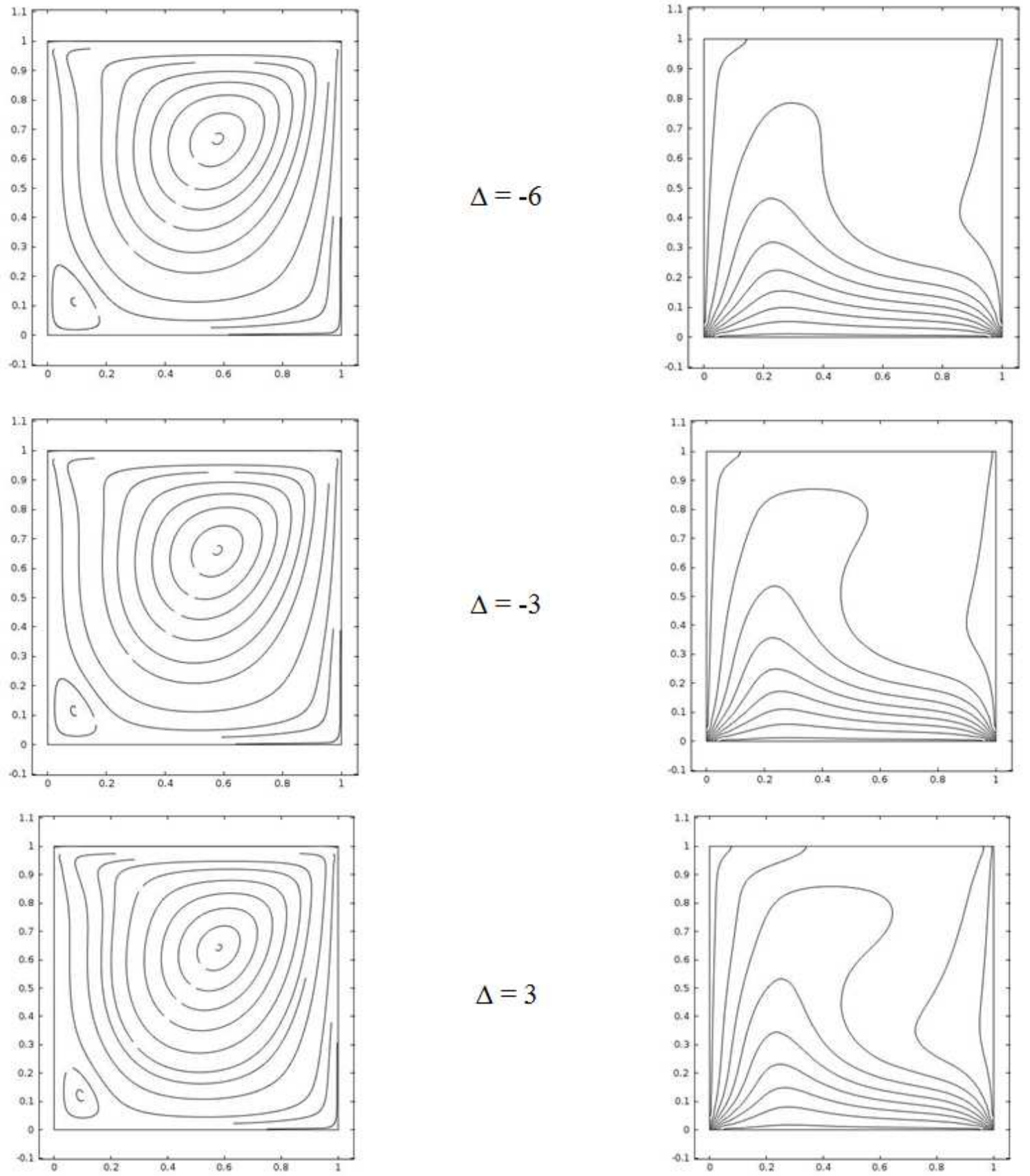

$\Delta=-3$

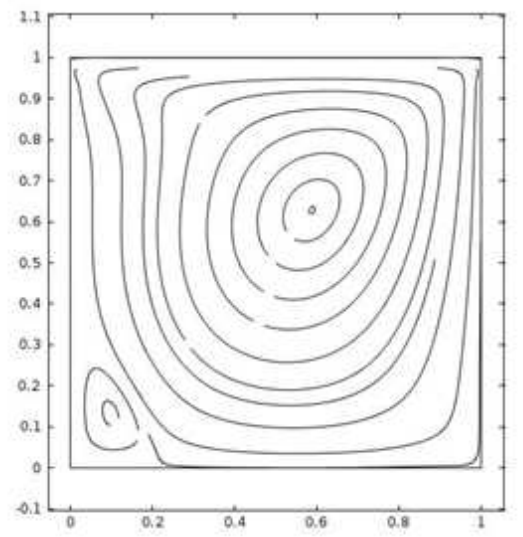

$\Delta=3$
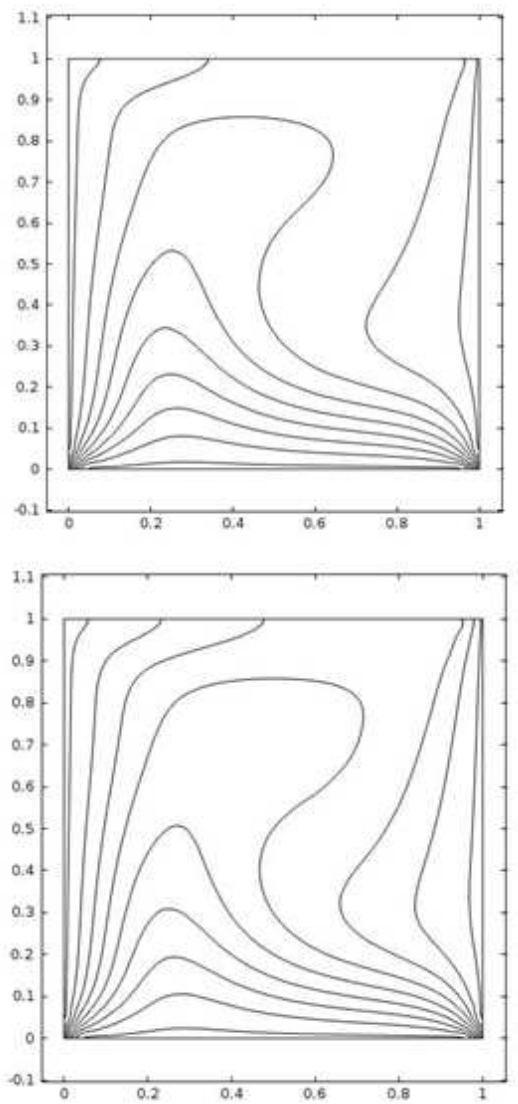

Fig. 3. Effect of $\Delta$ on the streamlines contour(left side) and temperature contour(right side) for $\mathrm{Ha}=0, \mathrm{Ri}=1$ and $\mathrm{Pr}=0.7$ 

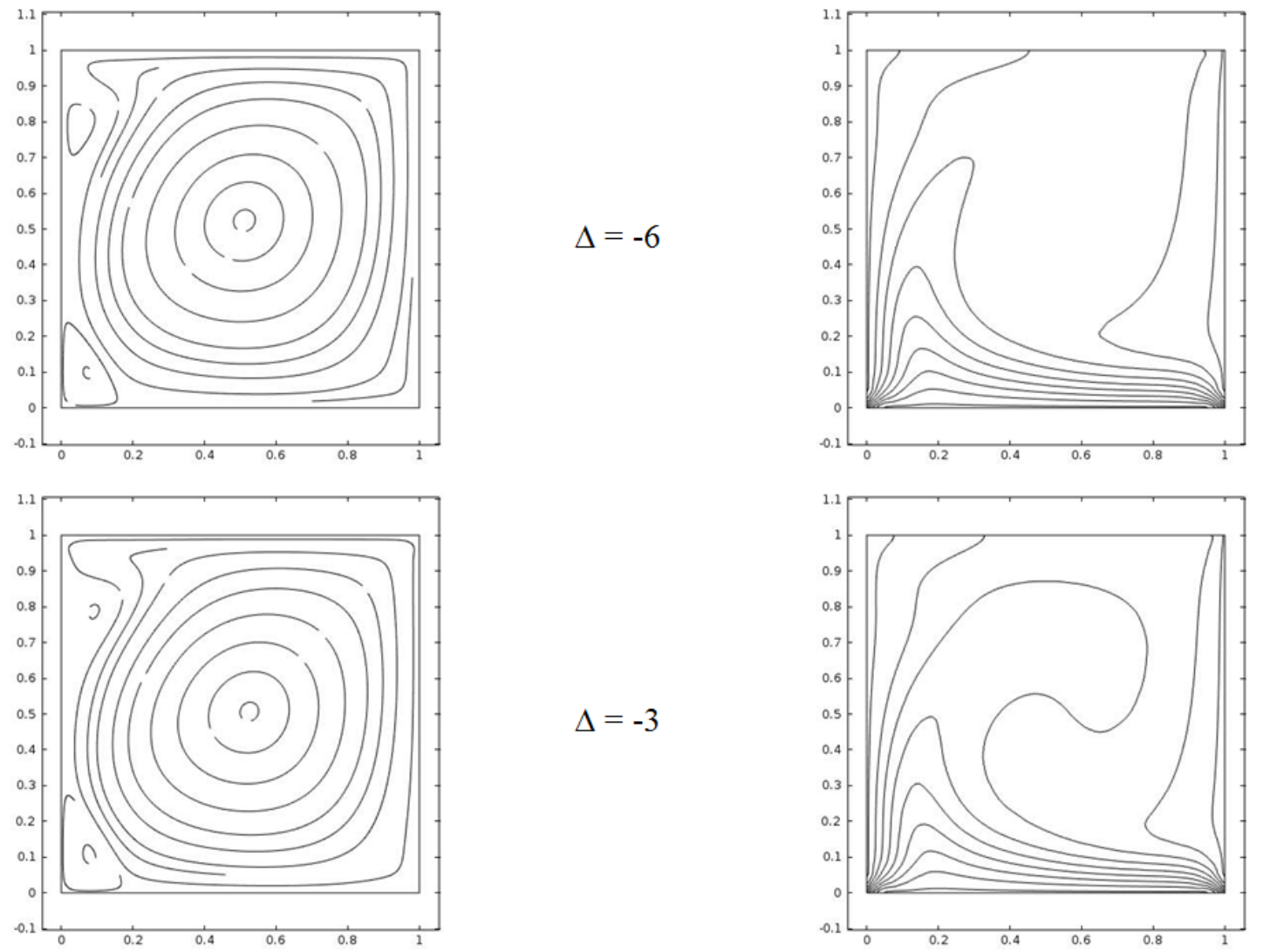

$\Delta=-6$

$\Delta=-3$
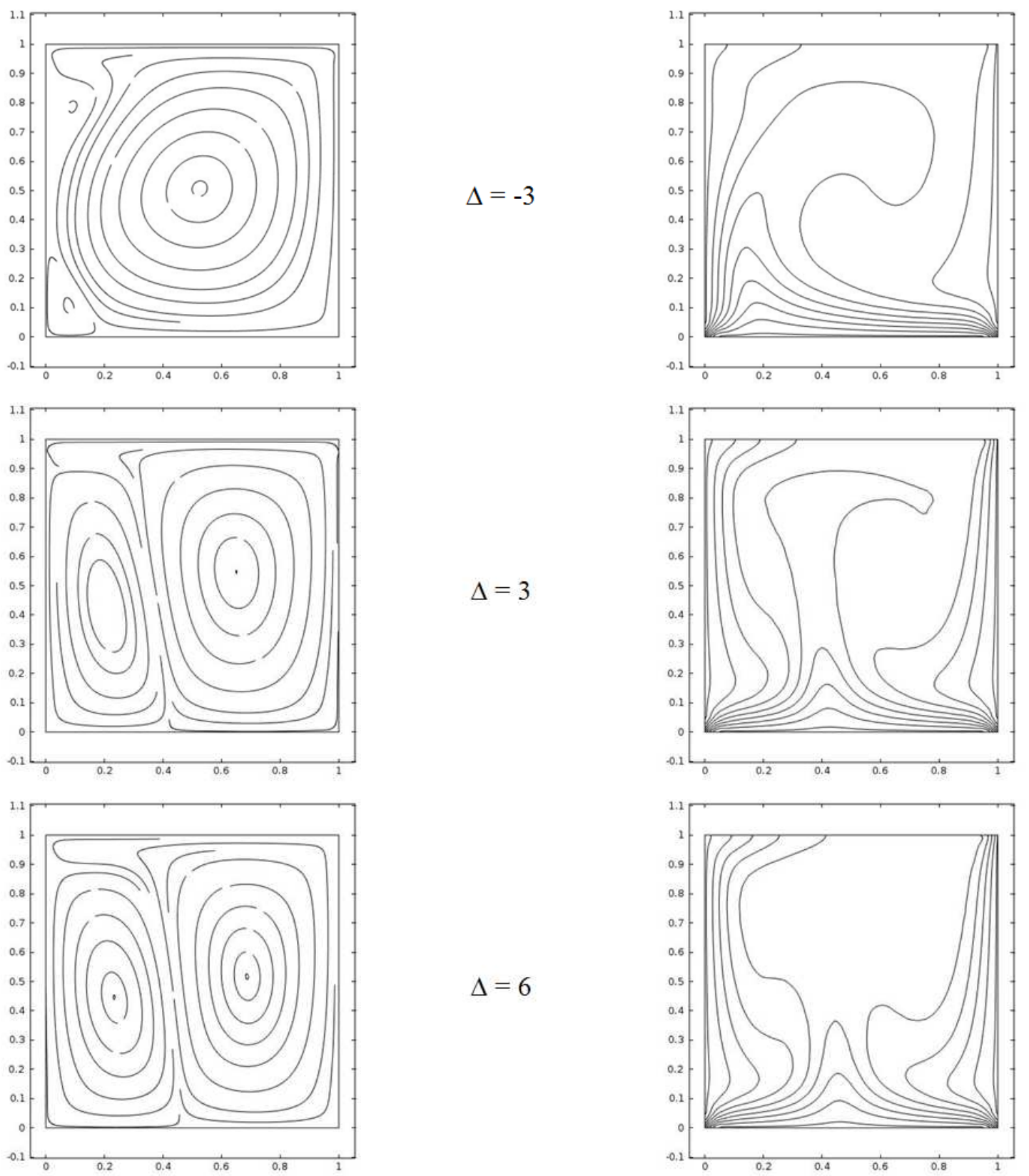

Fig. 4. Effect of $\Delta$ on the streamlines contour (left side) and temperature contour (right side) for $\mathrm{Ha}=0, \mathrm{Ri}=10, \mathrm{Pr}=0.7$ 

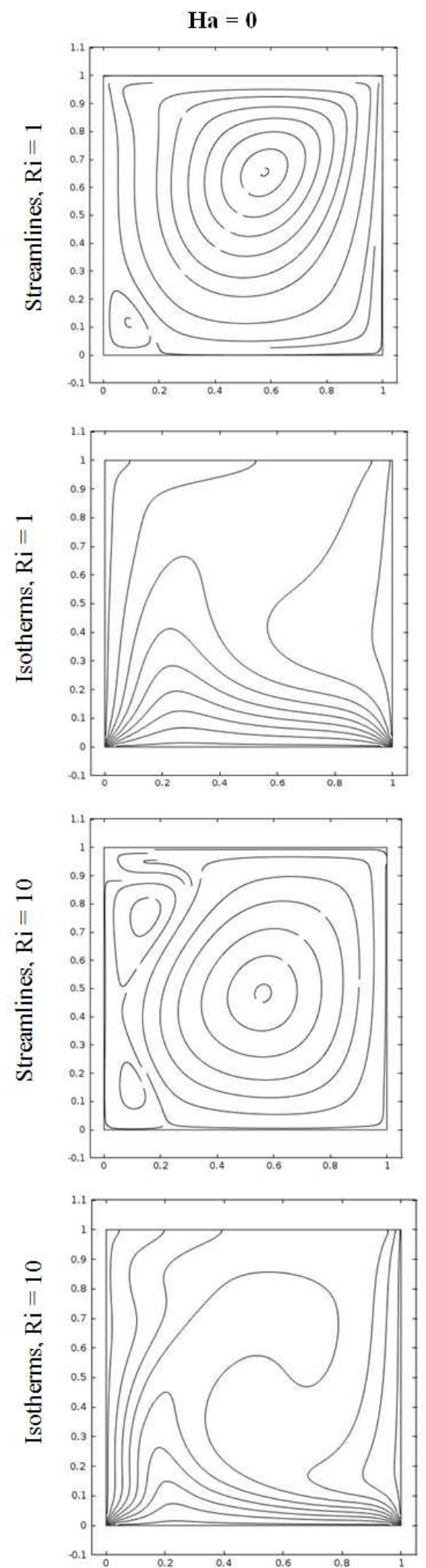

$\mathrm{Ha}=\mathbf{5 0}$

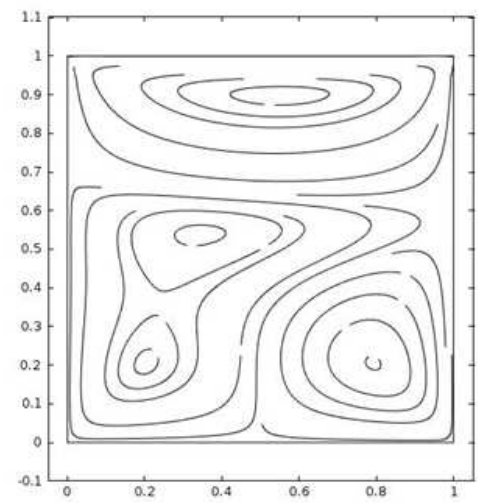

$\mathrm{Ha}=100$
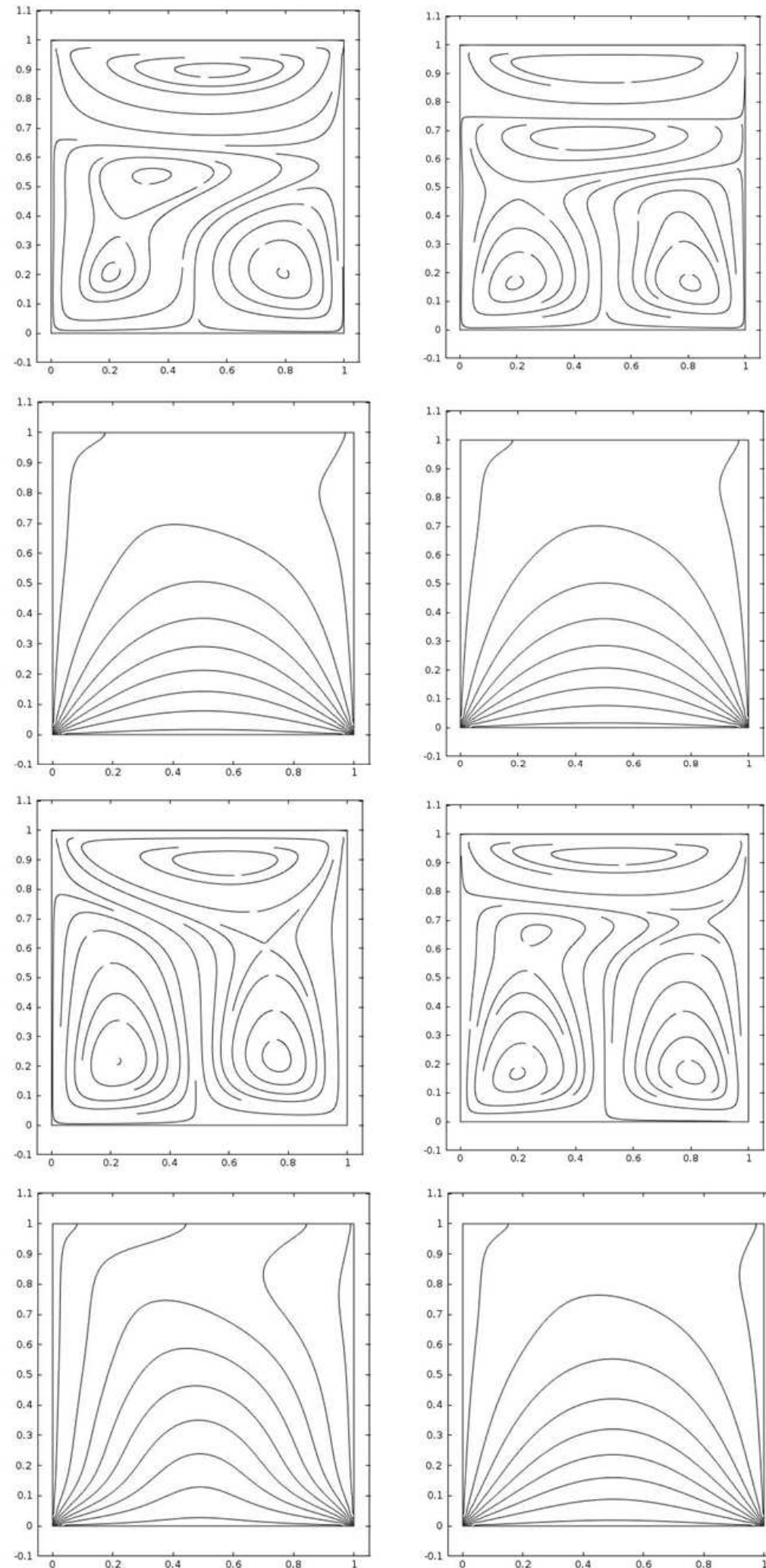

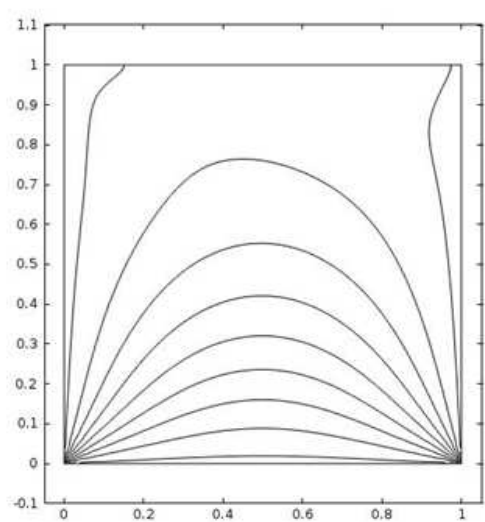

Fig. 5. Effect of Ha on the streamlines contour and temperature contour for $\Delta=0, P r=0.7, R i=1$ and 10

\section{Results and Discussions}

The characteristics of the flow and temperature fields in the lid driven cavity are examined by exploring the effects of the Richardson number Ri, which is the ratio of Grashof number and square of Reynolds number i.e., $\mathrm{Gr} / \mathrm{Re}^{2}$, the Hartmann 
number Ha and the heat generation or absorption coefficient $\Delta$. Such field variables are examined by outlaying the steady state version of the streamlines and temperature distributions as well as the average Nusselt number $\mathrm{Nu}$. In the current numerical investigation, the following parametric domains of the dimensionless groups are considered: $0.1 \leq \mathrm{R} \boldsymbol{i} \leq 10,0 \leq \mathrm{Ha}$ $\leq 100,-6 \leq \Delta \leq 6$ and $\operatorname{Pr}=0.7$.

The effects of the internal heat generation or absorption coefficient $\Delta$ on the contour maps of temperature and streamlines for $\mathrm{Re}=100, \mathrm{Gr}=10^{4}$ and $10^{5}$, which corresponds to the values of Richardson number $\mathrm{Ri}=1$ and 10 , are shown in Figure 3 and 4 respectively. As seen from Fig. 3, the streamlines contour remain almost unchanged for all values of $\Delta(-6,-3,36)$ in the case of $\mathrm{Ri}=1$ which indicates that the natural convection flow is quite weak to influence the flow field significantly.
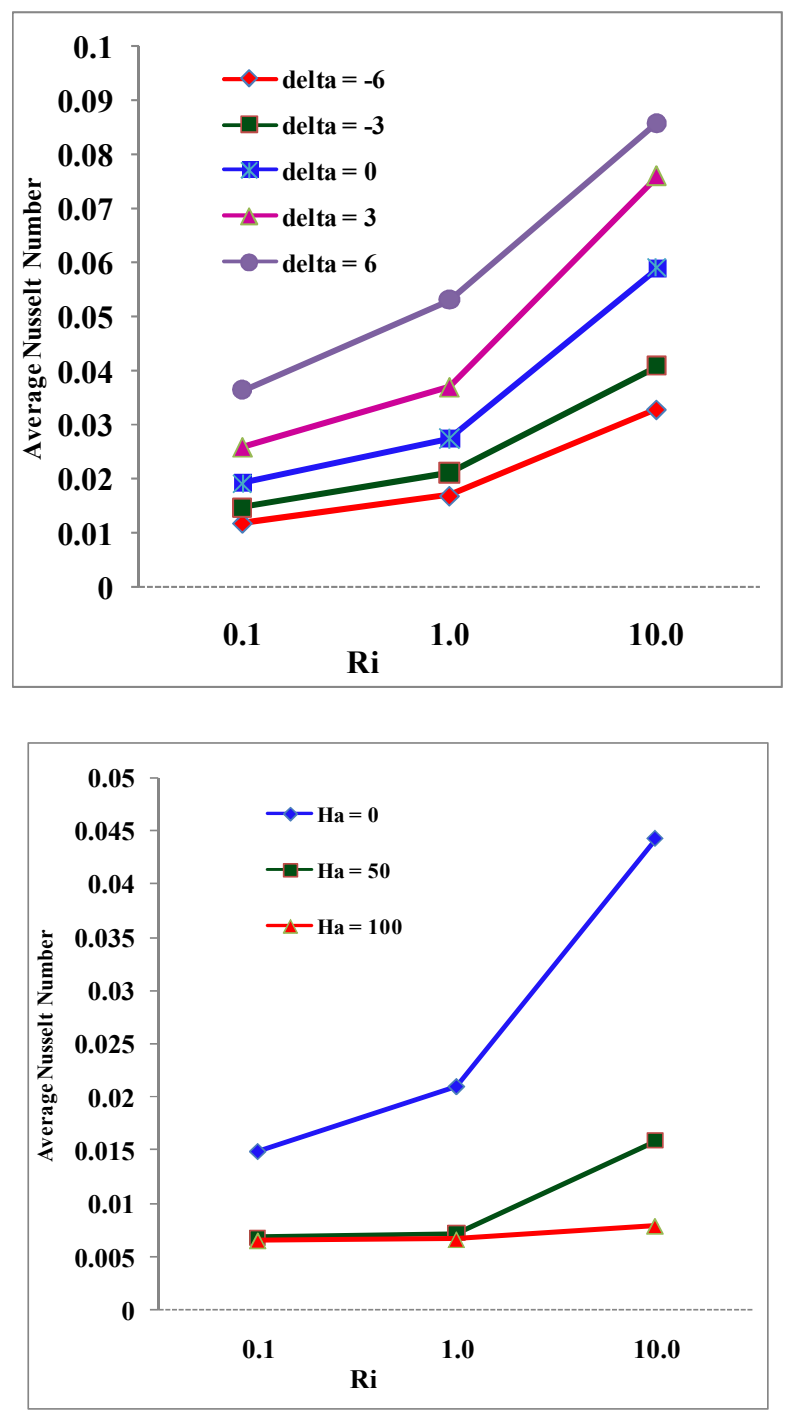

Fig. 6. Effect of $\Delta$ and Ha on the Average Nusselt numbers along the upper wall of the cavity for various Richardson number

Therefore, the flow due to driving lid plays dominant role than that of buoyancy forces. Hence the flow field and remains almost same for all values of $\Delta$. As a result, the role of internal heat absorption/generation on the resultant flow field remains insignificant. For a fixed values of Re, increasing values of $\mathrm{Gr}$ increases the values of Richardson number. In the case of $\mathrm{Ri}=10$, the effect of heat generation/absorption becomes very important. As the values of $\Delta$ increases, flow field undergoes significant changes. The secondary minor vortices become dominant the flow strength in this new cell also increases when the internal heat generation increases in magnitude as shown in Fig 4. For the case of internal heat absorption, flow field becomes more stabilized and minor vortices disappear gradually by the increasing values of heat absorption parameter. In contrast to the streamlines, the isotherms inside the cavity are found to undergo some changes due to the effect of internal heat generation/absorption even in the case of $\mathrm{Ri}=1$. As the internal heat generation effect $(\Delta>0)$ increases a localized region of high temperature is noticed in the core region and the high temperature region is extended toward the top adiabatic wall. This is due to the fact that the hot fluid cannot reject energy near the top adiabatic wall. This effect becomes clearer for the increasing values of Richardson number. For the case of internal heat absorption, opposite phenomena happens where the high temperature region moves closer to the hot base wall. It may be noted that on the right wall, the buoyancy and shear forces are in the same direction whereas on the left wall they are opposing each other. Less energy is noticed to be carried away from the sliding top wall into the cavity and, subsequently, the conduction heat transfer regime has become the dominant mode of energy transport in the cavity as a result a strong thermal boundary layer develops near the bottom and left wall

The effect of varying Hartmann number $\mathrm{Ha}$ on the streamlines contour and isotherms in a cavity for $\mathrm{Ri}=1$ and 10 is presented graphically in Figs 5. When the buoyancy effect is comparable to the effect of heated sliding wall, i.e. $\mathrm{Ri}=1$, the streamlines contour over the whole cavity region is occupied by a single primary vortex and a minor vortex at the left corner of the cavity for the values of Hartmann number $\mathrm{Ha}=0$. The primary vortex become smaller and more cells develops at lower portion of the cavity and it gradually becomes enlarger for the increasing values of Hartmann number. The flow strength decreases with increasing values of Hartmann number. In absence of Hartmann number, isotherms shows asymmetric pattern and heat is transferred by conduction and convection. Isotherms become symmetric with the increasing values of $\mathrm{Ha}$ and heat transfer is limited by conduction mode only. Thermal boundary layer increases with increasing of Hartmann number. The influences of the heat generation/absorption parameter $\Delta$ and the Hartmann number Ha on the average Nusselt number for various Richardson number Ri are graphically established in Fig. 6(a)-(b). From figure 6(a) it is observed that for a fixed value of the Re, increases in the value of the $\mathrm{Gr}$ (that is, increases in the value of the Richardson number $\mathrm{Gr} / \mathrm{Re}^{2}$ ) have the tendency to increase the convection heat transfer causing increases in the average Nusselt number, irrespective of the values of internal heat generation/absorption $\Delta$. Moreover, heat generation is predicted to decrease the average Nusselt number while 
average Nusselt number increase more and more for the increasing values of heat absorption. The effects of Hartmann number on average Nusselt number $\mathrm{Nu}$ at the upper wall of the cavity is shown in the Fig. 6(b). The results exhibit that the average Nusselt number is found to increase for increase in $\mathrm{Ri}$, for all values of Hartmann number. It is also observed from the Fig. 6(b) that significant reduction of average Nusselt number found for the increasing values of $\mathrm{Ha}$ and the effect is prominent in the convection dominant region.

\section{Conclusion}

In the present paper a problem on mixed convection flow of an electrically conducting fluid filled in a cavity under an externally imposed uniform magnetic field including the additional effect of internal heat generation has been investigated numerically. The governing equations are solved by using the Galerkin finite element method. Comparisons with previously published work on special cases of the problem were performed and found to be in good agreement. It was found that the heat transfer mechanisms and the flow characteristics inside the cavity are strongly dependent on the Hartmann number, heat generation/absorption coefficient and Richardson number. Effect of heat generation/absorption becomes insignificant for forced convection dominated region while it has significant effect for higher values of Ri. Flow strength decreases and isotherms becomes symmetric for the increasing values of Ha.

Finally, Heat transfer rate decreases with increasing of Hartmann number and heat generation parameter whereas increases for the increasing values of heat absorption parameter. Thus, magnetic field plays an important role to control heat transfer and fluid flow. The rate of reduction is higher for high values of the Richardson number.

\section{References}

[1] Koseff JR, Prasad AK. The lid-driven cavity flow: a synthesis of quantitative and qualitative observations. J Fluids Eng Trans ASME 1984;106:390-8.

[2] Morzinski M, Popiel CO. Laminar heat transfer in a two-dimensional cavity covered by a moving wall. Numer Heat Transfer 1988;12:265-73.

[3] Nasrin R., Parvin S., Hydromagnetic effect on mixed convection in a lid-driven cavity with sinusoidal corrugated bottom surface, International Communications in Heat and Mass Transfer 38 (2011) 781-789

[4] A.A. Mohamad, R. Viskanta, Flow and heat transfer in a lid-driven cavity filled with a stably stratified fluid, Appl. Math. Modelling 19 (1995) 465472.

[5] L. Fuchs, L.N. Tillmark, Numerical and experimental study of driven flow in a polar cavity, Int. J. Numer. Methods Fluids 5 (1985) 311-329.

[6] C. Migeon, A. Texier, G. Pineau, Effects of lid driven cavity shape on the flow establishment phase, J. Fluids Struct. 14 (2000) 469-488.
[7] M.K. Moallemi, K.S. Jang, Prandtl number effects on laminar mixed convection heat transfer in lid-driven cavity, Int. J. Heat Mass Transfer 35 (1992) 1881-1892.

[8] A. Al-Amiri, K. Khanafer, J. Bull, Ioan Pop, Effect of sinusoidal wavy bottom surface on mixed convection heat transfer in a lid-driven cavity, International Journal of Heat and Mass Transfer 50 (2007) 1771-1780.

[9] M.H. Chang, C.H. Cheng, Predictions of lid-driven flow and heat convection in an arc-shape cavity, Int. Commun. Heat Mass 26 (1999) 829-838.

[10] C.L. Chen, C.H. Cheng, Experimental and numerical study of mixed convection and flow pattern in a lid-driven arc-shape cavity, Heat Mass Transfer 41 (2004) 58-66.

[11] C.H. Cheng, C.L. Chen, Numerical study of effects of inclination on buoyancyinduced flow oscillation in a lid-driven arc-shaped cavity, Numer. Heat Transfer A 48 (2005) 77-97.

[12] L. K. Saha, M. C. Somadder, K. M. Salah Uddin, Mixed convection heat transfer in a lid driven cavity with wavy bottom surface, American Journal of Applied Mathematics, 2013; 1(5): 92-101

[13] H.F. Oztop, Z. Zhao, B. Yu, Conduction-combined forced and natural convection in lid-driven enclosures divided by a vertical solid partition, Int. Commun. Heat Mass Transf. 36 (2009) 661-668.

[14] K. Khanafer, A.J. Chamkha, Mixed convection flow in a lid-driven enclosure filled with a fluid-saturated porous medium, Int. J. Heat Mass Transfer 42 (1999) 2465-2481.

[15] S.K. Mahapatra, A. Sarkar, A. Sarkar, Numerical simulation of opposing mixed convection in differentially heated square enclosure with partition, Int. J. Therm. Sci. 46 (2007) 970-979.

[16] M. A. Hossain, M. Z. Hafiz, D. A. S. Rees, Buoyancy and thermocapillary driven convection flow of an electrically conducting fluid in an enclosure with heat generation, International Journal of Thermal Sciences 44(2005) 676-684

[17] J. M. Lee, M. Y. Ha and H. S. Yoon, Natural convection in a square enclosure with a circular cylinder at different horizontal and diagonal locations, Int. J. Heat Mass Transfer, 53(2010) 5905-5919.

[18] R. Iwatsu, J. M. Hyun, K. Kuwahara, Mixed convection in a driven cavity with a stable vertical temperature gradient, Int. J. Heat Mass Transfer, 25, (1993) 1601-1608

[19] T. Basak, S. Roy, Pawan Kumar Sharma, I. Pop, Analysis of mixed convection flows within a square cavity with uniform and non-uniform heating of bottom wall, International Journal of Thermal Sciences 48(2009) 891-912

[20] Chandra, B. and Gosh, N. (2001), MHD flow of a viscoelastic liquid through porous $\neg$ medium, International Journal of Numerical Methods for Heat and Fluid, vol. 11, pp. 682-698

[21] A.J. Chamkha, Hydromagnetic combined convection flow in a vertical lid driven cavity with internal heat generation or absorption, Numer. Heat Transfer A 41 (2002) 529-546.

[22] J.P. Garandet, T. Alboussiere, R. Moreau, Buoyancy driven convection in a rectangular enclosure with a transverse magnetic field, International Journal ofHeat and Mass Transfer 35 (4) (1992) 741-748 
[23] M.M. Rahman, M.A. Alim, M.M.A. Sarker, Numerical study on the conjugate effect of joule heating and magneto-hydrodynamics mixed convection in an obstructed lid-driven square cavity, Int. Commun. Heat Mass Transfer 37 (2010) 524-534.

[24] H. F. Oztop K. Al-Salem, , I. Pop, MHD mixed convection in a lid-driven cavity with corner heater, International Journal of Heat and Mass Transfer 54 (2011) 3494-3504.

[25] N. Rudraiah, R.M. Barron, M. Venkatachalappa, C.K. Subbaraya, Effect of a magnetic field on free convection in a rectangular enclosure, Int. J. Eng. Sci. 33 (1995) 1075-1084.

[26] N. Al-Najem, K. Khanafer, M. El-Refaee, Numerical study of laminar natural convection in tilted enclosure with transverse magnetic field, Int. J. Numer. Methods Heat Fluid Flow 8 (1998) 651-672.

[27] K. Kahveci, S. Oztuna, MHD natural convection flow and heat transfer in a laterally heated partitioned enclosure, Eur. J. Mech. B Fluids 28 (2009) 744-752.

[28] C. Taylor, P. Hood, A numerical solution of the Navier-Stokes equations using finite element technique, Computers \& Fluids 1 (1) (1973) 73-89.

[29] P. Dechaumphai, Finite Element Method in Engineering, 2nd ed. Chulalongkorn University Press, Bangkok, 1999. 